\title{
Development of Highly Selective Interdigitated Electrode (IDE) Sensor Array using Molecular Imprinted Polymer (MIP) for Detection of Mango Fruit Ripeness
}

\author{
H. F. Hawari ${ }^{1}$, N. M. Samsudin ${ }^{1}$, A. Y. Md. Shakaff', Y. Wahab' ${ }^{2}$, U. Hashim², \\ A. Zakaria', S. A. Ghani , M. N. Ahmad ${ }^{1}$ \\ ${ }^{1}$ Centre of Excellence for Advanced Sensor Technology (CEASTech), Pusat Pengajian \\ Jejawi II, Taman Muhibbah, University Malaysia Perlis, 02600 Arau, Perlis, Malaysia. \\ huzeinfahmi@gmail.com \\ ${ }^{2}$ Inst. Nano Electronic Eng, University Malaysia Perlis, Malaysia.
}

\begin{abstract}
:
An electronic nose using of MIP (Molecularly Imprinted Polymer) array is developed to give a unique fingerprint of mango volatiles when ripe. Volatiles such as $\alpha$-pinene, $\gamma$-terpinene and terpinolene are found to be released during different stages of fruit maturity. Utilizing these different volatiles, MIPs templates were then synthesized and deposited onto the Interdigitated Electrode (IDE) platform to acquire a specific fingerprint of fruit maturity. It was found that the best composition ratio (Template: MAA: EGDMA) for IDE MIP Sensor is $(1: 5: 20)$. When exposed to a specific mango volatiles, the IDE-MIP sensor array exhibited a specific sensitivity and high selectivity. These studies provide a potential solution for agriculture sector to improve the quality of harvest.
\end{abstract}

Key words: Molecularly Imprinted Polymer, electronic nose, sensor array, mango volatiles, fruit ripeness.

\begin{abstract}
Introduction
Determining the right time for a fruit ripe enough to harvest is one of the most important technological aspects in the agriculture industry. Traditionally, fruit ripeness has been performed through traditional harvesting method which are based on smell, texture and harvesting time [1, 2]. This analytical technique has many disadvantages in terms of objectivity and subjectivity. By determining various volatile compound released during pre-matured until matured period, one can actually determine the ripeness stage thus contributing to an optimal harvesting.
\end{abstract}

Throughout the years, extensive research has been done to study the major volatiles present during the maturity stages of mango. Differences volatile compounds produced during normal mango ripening have been reported. It was found that terpene hydrocarbons are the major class of compounds in mango [3]. For a ripened "Cogshall' mango, $\alpha$-pinene, $\beta$-pinene, $\alpha$ terpinolene, $\gamma$-terpinene, and $\beta$-caryophyllene are found to be released at higher levels of most volatiles [4] while for 'Kensington Pride' mango, $\alpha$-Terpinolene was the major compound during the first 7 days of ripening [5]. Figure 1 shows chemical structure of the 3 template used in this research.

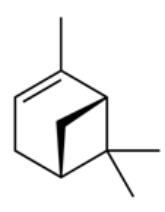

a-pinene

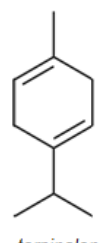

terpinolene

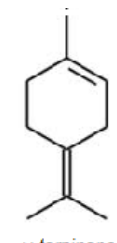

$\gamma$-terpinene
Fig. 1. Structure of volatiles used for molecular imprinting

Molecular Imprinted Polymer (MIP) is an established method targeted for the production of polymeric artificial receptors of specific molecular recognition. Apart from inexpensive and are highly stable, molecular imprinting has also been used to produce a large variety of materials which have selective adsorption properties for specific molecules, $[6,7,8]$. The imprinting molecule was used as a template to form a receptor site with complementary 
binding features with regard to the molecule's shape, structure, and spatial orientation of the peripheral functionality. Basic concept of MIP is illustrated in Figure 2 below. Removal of templates will then leave cavity that is selective to template molecule.

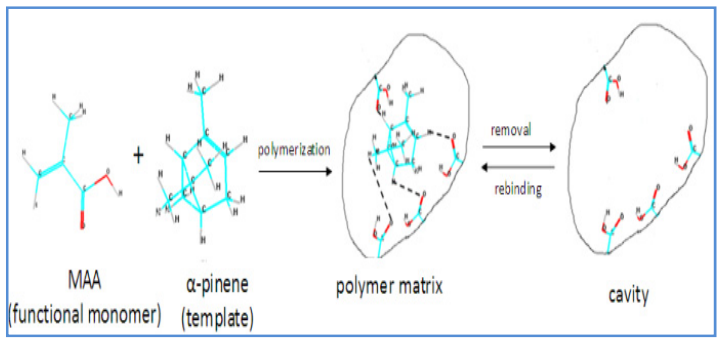

Fig. 2. Basic Concept of MIP

Electronic nose (E-nose) is an artificial olfaction device that is able to replicate the human olfactory sense. Within this work, we focus on the development of a molecularly imprinted polymer (MIP)-based Interdigitated Electrodes (IDE) sensor array which are specifically designed to respond to the surrounding odorants or volatiles.IDE are made up by two overlapping metal comb structure. Each metal comb shall act as one electrode. The MIP is then deposited on the free opposite side and has direct contact to the comb electrode. In order to identify the capacitance, $\mathrm{C}$ value of a simple parallel plate capacitor structure the capacitance is given by

$$
C=\frac{\varepsilon_{0} \varepsilon_{\mathrm{r}} \mathrm{A}}{d}
$$

where $\varepsilon_{r}$ is the dielectric constant of the material between the plates, $\varepsilon_{0}$ is the dielectric constant for a vacuum, $A$ is the area and $d$ the distance between the plates. The capacitance changes because of adsorption or desorption of MIP. Since capacitive measurements give information about the metal solution interface, a chemical modification of this structure will lead to a change in capacitance, thereby acting as a sensor.

\section{Materials and Methods}

Interdigitated Electrodes Fabrication

In this research, a gold IDE with dimensions of $5 \mathrm{~mm} \times 10 \mathrm{~mm}$ is used as a sensor. The IDE is fabricated through sputtering machine with thickness of 292A thin films of gold onto a Polyethylene Perephthalate (PET) substrate. PET is used for this research due to it's flexiblity but very low in cost. For this research, an eight finger metal mask was used to sputter the gold IDE. As in figure 3, both the spacing between the mask fingers and their width were equal to 500 micron.

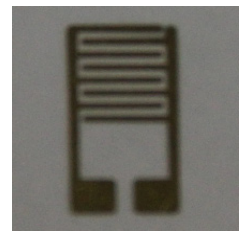

Fig. 3. IDE with 500 micron gap size.

\section{Fruit and ripening conditions}

Similarly, we have selected Harumanis mango (Mangifera Indica. $L$ ) grown in Perlis (one of the state in Northern Malaysia) to study the volatile released during different stage of mango maturity. Fresh mature and ripe mangoes were selected from a batch of single harvest by the state agriculture department located in Bukit Temiang, Perlis for international export.

\section{Chemicals/Materials}

Terpinolene, $\alpha$-pinene, $\mathrm{y}$-terpinene, Methacrylic Acid (MAA), Ethylene Glycol Dimethacrylate Acid (EGDMA), 2-Aminoethanethiol (AET) were purchased from Acros Organics, Belgium. Tetrahydrofuran (THF) was purchased from Fisher Scientific, U.K while PET was purchased from Sigma Aldrich.

\section{Cross-linker}

A cross-linker is added to fulfill three major functions in imprinting. High cross-link ratios are generally preferred to access permanently porous materials and to generate mechanical stability of the materials. Therefore, polymer with high degree of cross-linking $(70-90 \%)$ is required. In this research, Ethylene Glycol Dimethacrylate (EGDMA) was used with the ratio of 20:1 (EGDMA:template) approximately $3.77 \mathrm{ml}$

\section{Functional Monomer}

Choosing the functional monomer is a crucial part in synthesizing the MIP since it is responsible for the binding interactions in the imprinted binding sites. The exact value of functional monomer ratio can be predicted by applying computational modelling thus eliminating timely trial and error experiment to predict the optimum ratio of functional monomer to bind with template molecules. In this study, methacrylic acid (MAA) was used as functional monomer and the ratio of functional monomer was predicted by the binding energy of interaction between complexes (templateMAA). 
Initiator

The purpose of adding initiator to the MIP is to initiate free radical polymerization. When the initiator is trigged by UV radiation or heat, carbon centre free radicals will formed. These unpair electrons are capable to react with other monomer/cross-linker to propagate longer chains. AIBN (Azobisisonutyronitrile) was used as initiator in this study and the amount used is $0.01 \mathrm{~g}$ per sample.

\section{Preparation of MIP Solution}

One mmol template $\alpha$-pinene $(0.1603 \mathrm{ml})$ was added to $5 \mathrm{mmol}(0.4262 \mathrm{ml})$ functional monomer (MAA) and stirred together with $0.01 \mathrm{~g}$ initiator AIBN. The mixture was dissolved in $10 \mathrm{ml}$ THF. Twenty mmol $(3.776 \mathrm{ml})$ EGDMA was added to the mixture and stirrer for 5 minutes [9]. The mixture was purged with nitrogen for 3 minutes to remove oxygen. Similar procedure was repeated for other templates ( $\gamma$-terpinene, $\alpha$-pinene and terpinolene). Non-Imprinted polymer (NIP) was also prepared and treated the same manner as MIP for control purpose.

\section{Results and discussion}

Gas Chromatography Mass Spectroscopy (GCMS) analysis on Harumanis Mango

Harumanis mango with difference maturity, mature and ripe are placed in desiccators with 60 minutes incubation and 15 minutes Solid Phase Microextraction (SPME) fiber exposure. From figure 4 , analysis of volatiles by GC-MS showed evidence of volatiles such as a-pinene, 3-carene, cedrene and $y$-terpinene present in a ripe mango compared to a mature mango. Identification of all aromatic components was achieved by means of Clarus 600 Gas Chromatography /Mass Spectrometer (Perkin Elmer, USA)
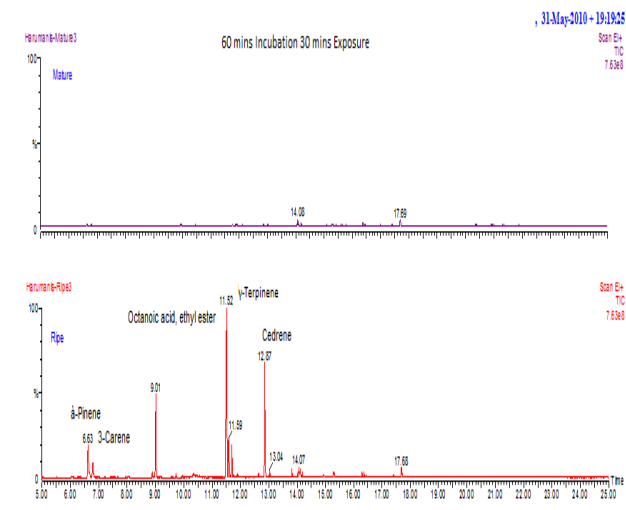

Fig. 4. GC-MS result on Harumanis mango with difference maturity- mature and ripe
Fourier Transform Infrared Spectroscopy (FTIR) analysis

In this research, FTIR analysis is used as preliminary determination of complex (templatefunctional monomer) interaction MIP. This is useful to predict stability and interaction capacity of recognition cavities towards template molecules and detection of the efficacy extraction and rebinding procedures [10].

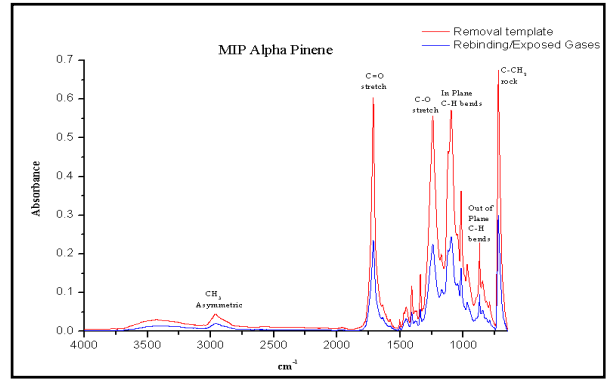

Fig. 5. FTIR peak comparison of removal and rebinding stages.

In figure 5, the strongest absorption peak is investigated at removal of templates peak (red color) and the blue peak is the rebinding of template molecules. The $\mathrm{CH}_{3}$ Asymmetric at $2961.38 \mathrm{~cm}^{-1}$ (removal) and $2958.39 \mathrm{~cm}^{-1}$ (rebinding) characterizes the $-\mathrm{CH}_{3}$ group of skeleton. The carboxylate group $\left(\mathrm{COO}^{-}\right)$is well evinced by characteristic absorption stretch at $1713.13 \mathrm{~cm}^{-1}$ (removal) and $1712.27 \mathrm{~cm}^{-1}$ (rebinding) with the absorption of 0.6025 , and 0.2381 respectively. The high absorption during removal is due to the functional monomer MAA (contain carboxyl group) that ready to absorb and bind the template (a-pinene). MAA was choose die to the presence of $\mathrm{COOH}$ group that commonly hydrogen bonding with the template. The characteristic of $\mathrm{C}-\mathrm{H}$ bond was observed at $1096.68 \mathrm{~cm}^{-1}$ and $871.56 \mathrm{~cm}^{-1}$ during removal and $1096.21 \mathrm{~cm}^{-1}$ and $871.59 \mathrm{~cm}^{-1}$ during rebinding. Lastly, $\mathrm{C}-\mathrm{CH}_{2}$ bond was observed at high absorption during removal. $\mathrm{C}-\mathrm{CH}_{2}$ characterized the skeleton which contains double bond. It was observed at 722.14 (removal) $\mathrm{cm}^{-1}$ and $722.35 \mathrm{~cm}^{-1}$ (rebinding) with high stretch of absorption at removal stage of 0.6725 compared to 0.3026 for rebinding.

IDE and measurement

Using Alpha-N High Resolution Dielectric Analyzer (Novocontrol, Germany) with Windeta software, the capacitance value of the IDE in the air was measured to be approximately of 1$3 \mathrm{pF}$. The tested frequency range was from 0.1 $\mathrm{kHz}$ to $1 \mathrm{kHz}$, with an alternating potential voltage of $100 \mathrm{mV}$. After template removal, the 
difference in capacitance before and after the mango volatile binding was taken as the signal produced by reaction between the MIP and volatile. The signal response toward the mango analytes of the sensor was reported as $\Delta C(p F)$, where $\Delta \mathrm{C}$ is the capacitance shift in response to the addition of known amounts of the volatile of interest. In figure 6, IDE MIP sensor array consist of 3 IDE MIP of $\alpha$-pinene, terpinolene and $\mathrm{Y}$-terpinene are exposed to $\alpha$-pinene volatile. It is found that the $\Delta C$ of $\alpha$-pinene IDEMIP is the highest among 3 IDE MIP sensors followed by $\mathrm{y}$-terpinene and terpinolene IDEMIP sensor.

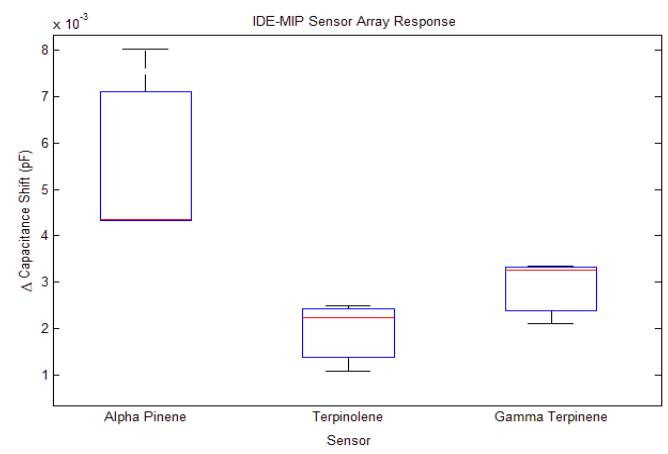

Fig. 6. Sensor array response when exposed to a- pinene volatiles

\section{Conclusion}

In this research, a sensor array to detect mango volatiles was successfully implemented by using MIP as the sensing materials. Through analysis by GC-MS, concentration of volatiles such as a-pinene, 3-carene, cedrene and $y$ terpinene are found higher in a ripe mango compared to a matured mango. The sensitivity of IDE MIP sensor is influenced by preparation of MIP solution. It was found through computer modelling the best composition ratio for template: MAA: EGDMA is 1:5:20. Through FTIR study, it is found that the strongest absorption peak is due to carboxyl group that ready to absorb and bind with the template. An IDE is used as a platform for detection of electrical capacitance due to modification of the dielectric constant of the MIP after binding with mango volatile. When exposed to specific mango volatiles, the IDE-MIP sensor array exhibited a specific sensitivity and selectivity of sensor responses. Apart from that, the sensor is miniature in size with flexible substrate and can be used repeatedly after template removal. Additional efforts is currently in progress to explore more mango volatiles and array combination in order to further enhance the IDE MIP sensor selectivity in detecting mango ripeness.

\section{Acknowledgements}

We wish to thank Ministry of Higher Education Malaysia for their financial support (FRGS 9003-00250) of H.F. Hawari and N.M Samsudin during their studies.

\section{References}

[1] C.M. Christensen, Effects of color on aroma, flavor and texture judgements of foods, J. Food Sci. 48, pp. 787-790(1983); doi:10.1111/j.13652621.1983.tb14899.x

[2] M.J.W. Povey, Ultrasonics in food engineering: Part II. Applications, J. Food Eng. 9, pp. 1-20 (1989) ; doi:10.1016/0260-8774(89)90047-2

[3] MacLeod, A. J.; MacLeod, G.; Snyder, C. H. Volatile aroma constituents of mango (cv Kensington). Phytochemistry 27 pp. 2189-2193. (1988) ; doi:10.1016/0031-9422(88)80124-9

[4] Lebrun, M., Plotto, A., Goodner,K., Ducamp, M., and Baldwin., E. Discrimination of Mango Fruit Maturity by Volatiles using the Electronic Nose and Gas Chromatography. Postharvest Biology and Technology, 48, pp. 122-131,(2008); doi: 10.1016/j.postharvbio.2007.09.010

[5] Lalel, Herianus. J.D. and Singh, Zora and Tan, Soon Chye. Aroma volatiles production during fruit ripening of 'Kensington Pride' mango. Postharvest Biology and Technology 27 (3): 323336(2003); doi: 10.1016/S0925-5214(02)00117-5

[6] Yongqin L., Zhixing, L., Tianwei T., Wei,F., Peiyong, Q., \& Cong., L. Application of Molecular Dynamics Modelling for the Prediction of Selective Adsorption Properties of Dimethoate Imprinting Polymer. Sensors and Actuators $B$ 133, pp. 15-23 (2008); doi: 10.1016/j.snb.2008.01.067

[7] Merkoci A, Alegret S, New materials for electrochemical sensing IV. Molecular imprinted polymers. Trends Anal Chem 21:717-725 (2002); doi: 10.1016/S0165-9936(02)01119-6

[8] Ye, L.; Haupt, K. Molecularly Imprinted Polymers as Antibody and Receptor Mimics for Assays, Sensors and Drug Discovery. Anal. Bioanal. Chem. 378, 1887-1897. (2004) ; doi: $10.1007 / \mathrm{s} 00216-003-2450-8$

[9] Huzein Fahmi Hawari, Nurul Maisyarah Samsudin, Mohd Noor Ahmad, Ali Yeon Md Shakaff, Supri. A. Ghani, Yufridin Wahab, Uda Hashim, "Recognition of Limonene Volatile Using IDE Molecular Imprinted Polymer Sensor," Third International Conference on Intelligent Systems Modelling and Simulation(ISMS), pp.723-726, (2012);doi:http://doi.ieeecomputersociety.org/10.1 109/ISMS.2012.103

[10] Tarannum, N., and Singh, M. Selective Recognition and Detection of L-Aspartic Acid by Molecularly Imprinted Polymer in Aqueous Solution, American Journal of Analytical Chemistry 2, pp. 909-918(2011); doi: 10.4236/ajac.2011.28105 